University of South Carolina

Scholar Commons

2005

\title{
Effects of Dynamic Fluid Pressure on Chondrocytes Cultured in Biodegradable Poly(glycolic acid) Fibrous Scaffolds
}

Lichun Lu

Xun Zhu

Larry G. Pederson

Esmaiel Jabbari

University of South Carolina - Columbia, jabbari@mailbox.sc.edu

Bradford Currier

See next page for additional authors

Follow this and additional works at: https://scholarcommons.sc.edu/eche_facpub

Part of the Chemical Engineering Commons

Publication Info

Published in Tissue Engineering Part A, Volume 11, Issue 11/12, 2005, pages 1852-1859.

(C) Tissue Engineering 2005, Mary Ann Liebert.

Lu, L., Zhu, X., Pederson, L.G., Jabbari, E., Currier, B.L., O'Driscoll, S., \& Yaszemski, M.J. Effects of Dynamic Fluid Pressure on Chondrocytes Cultured in Biodegradable Poly(glycolic acid) Fibrous Scaffolds. Tissue Engineering, 11(11-12). 1852-1859.

http://dx.doi.org/10.1089/ten.2005.11.1852

This Article is brought to you by the Chemical Engineering, Department of at Scholar Commons. It has been accepted for inclusion in Faculty Publications by an authorized administrator of Scholar Commons. For more information, please contact digres@mailbox.sc.edu. 


\section{Author(s)}

Lichun Lu, Xun Zhu, Larry G. Pederson, Esmaiel Jabbari, Bradford Currier, Shawn O'Driscoll, and Michael Yaszemski 


\title{
Effects of Dynamic Fluid Pressure on Chondrocytes Cultured in Biodegradable Poly(glycolic acid) Fibrous Scaffolds
}

\author{
LICHUN LU, XUN ZHU, LARRY G. PEDERSON, ESMAIEL JABBARI, \\ BRADFORD CURRIER, SHAWN O'DRISCOLL, and MICHAEL YASZEMSKI, M.D., Ph.D.
}

\section{INTRODUCTION}

C ARTILAGe Defects cause considerable pain for the individual patient and they inflict a heavy burden on society. An estimated 70 million Americans are affected by arthritis and related conditions and the total cost for treating these diseases is $\$ 82$ billion annually. ${ }^{1}$ The clinical problems range from small traumatic osteochondral defects to global destruction of the joint surface from arthritis. Damage to articular cartilage often requires surgical intervention because of its limited capacity for self-repair. The unique challenges of each clinical situation call for different treatment strategies. The current treatment options include shaving the articular surface, perforating the underlying subchondral bone, periosteal and perichondral grafts, chondrocyte transplantation, osteotomy, and artificial joint replacement. ${ }^{2}$ However, none of these techniques can effectively manage the symptoms and predictably restore normal cartilage function.

Tissue-engineering strategies using biodegradable polymers as scaffolds for cell transplantation provide many advantages over current therapies. The three components common to many cartilage tissue-engineering approaches are cells (either chondrocytes or chondroprogenitor cells), a scaffold, and various soluble and extracellular matrix signals. ${ }^{3,4}$ In addition to serving as a substrate, the scaffold can interact with the other elements in a dynamic and synergistic manner to direct and organize the process of regeneration.

Various synthetic materials have been explored to serve as scaffolds for cartilage tissue engineering. ${ }^{4,5}$ Injectable formulations based on poly(ethylene oxide), ${ }^{6}$ poly(anhydride), ${ }^{7}$ and oligo-poly(ethylene glycol)-fumarate $^{8}$ may be delivered through minimally invasive procedures to treat contained focal defects. Synthetic polymers as preformed scaffolds may be preferable for replacing large, noncontained defects. Among them, highly porous poly(glycolic acid) (PGA) nonwoven fibrous scaffolds have been used extensively for chondrocyte culture and subsequent transplantation. ${ }^{9-13}$ These studies have shown that PGA provides a suitable substrate for chondrocyte proliferation and cartilage tissue formation both in vitro and in vivo. However, the quality of the cartilage tissue formed in synthetic scaffolds is inferior to the natural cartilage in both structural organization and biomechanical properties.

The role of mechanical force in regulating chondrocyte function and maintaining normal cartilage tissue has long been recognized. ${ }^{14}$ During normal joint movement, cyclic forces generate complex and dynamic stress and strain fields throughout articular cartilage. At present, continuous passive motion (CPM) is used clinically in postoperative care after cartilage repair. ${ }^{15}$ CPM transmits forces to cartilage through direct deformation at the opposing cartilage surfaces in the knee joint as well as through normal and tangential fluid-induced forces. The focus of this study was on the role of hydrostatic force, the normal component of the fluidinduced forces experienced during CPM, on chondrocytes cultured in biodegradable polymer scaffolds. Specifically, we investigated the effects of dynamic fluid pressure (at a magnitude of $13 \mathrm{kPa}$ and a frequency of $0.3 \mathrm{~Hz}$ ) and the time course of pressurization $(4,5$, or 6 weeks of pressurization) on cultured chondrocyte-PGA constructs during a 6-week period. Low-level oscillations in hydrostatic pressure $(13 \mathrm{kPa}$ at $0.3 \mathrm{~Hz}$ ) were chosen because they are similar to the cyclic oscillations of intraarticular synovial fluid pressure during $\mathrm{CPM}^{16}$ and because cultured chondrocytes were shown to respond to such a mechanical stimulus. ${ }^{17}$ 


\section{MATERIALS AND METHODS}

\section{Articular cartilage harvest and chondrocyte isolation}

Articular cartilage was harvested aseptically from the femoropatellar grooves of 2-month-old New Zealand White rabbits. ${ }^{18}$ This protocol was approved by the Institutional Animal Care and Use Committee at the Mayo Clinic (Rochester, MN). Chondrocytes were isolated by digestion with a collagenase II solution (Worthington Biochemical, Lakewood, NJ). ${ }^{19}$ The primary cells were expanded in medium consisting of Dulbecco's modified Eagle's medium (DMEM), 10\% fetal bovine serum, $2 \mathrm{mM}$ L-glutamine, $0.1 \mathrm{mM}$ nonessential amino acids, L-proline $(50 \mu \mathrm{g} / \mathrm{mL}), 1 \mathrm{mM}$ sodium pyruvate, and gentamicin $(25 \mu \mathrm{g} / \mathrm{mL}$ ) (all from Invitrogen GIBCO, Grand Island, NY), as previously described. ${ }^{12}$

\section{Chondrocyte seeding and culture in polymer scaffolds}

Poly(glycolic acid) (PGA) nonwoven fibrous disks were purchased from Albany International Research (Mansfield, MA). These disks were $5 \mathrm{~mm}$ in diameter and $2 \mathrm{~mm}$ thick, with a fiber diameter of $13 \mu \mathrm{m}$, a void volume of $97 \%$, and a bulk density of $62 \mathrm{mg} / \mathrm{mL}$. Before use in cell culture, PGA disks were sterilized with ethylene oxide and prewetted by sequential immersion in $70 \%$ ethanol, phosphate-buffered saline (PBS; Invitrogen GIBCO), and chondrocyte culture medium (see Articular Cartilage Harvest and Chondrocyte Isolation, above).

Chondrocytes at first passage were seeded at a density of $5 \times 10^{6}$ cells per scaffold in the prewetted PGA disks placed in 12-well plates (non-cell culture treated; Fisher, Pittsburgh, PA) according to the method described by Schreiber et al. ${ }^{19}$ The cell-polymer constructs were cultured for a total of 6 weeks with the culture medium changed every 2 to 3 days. Insulin and ascorbic acid at $50 \mu \mathrm{g} / \mathrm{mL}$ were added at each feeding.

The chondrocyte-PGA constructs were divided into four sample groups, identified by culture condition ( $\mathrm{S}$, static; D, dynamic fluid pressure) and the number of weeks for each condition. After 1 day of cell seeding, the constructs were cultured for 6 weeks under static conditions (Static), 1 week of static culture and then 5 weeks under dynamic fluid pressure (S1/D5), 2 weeks of static culture and then 4 weeks under dynamic fluid pressure (S2/D4), or 6 weeks under dynamic fluid pressure (DFP). During the fluid pressure culture period, samples were pressurized at a magnitude of $13 \mathrm{kPa}$ and a frequency of $0.3 \mathrm{~Hz}$ for a total of $4 \mathrm{~h} /$ day. All constructs were placed in the pressure chamber for $4 \mathrm{~h} /$ day and all cells were allowed to experience the purge cycle.

\section{Application of dynamic fluid pressure}

Dynamic fluid pressure was applied to the chondrocyte-PGA constructs by compression of the gas phase above the culture medium covering the constructs, using a custom-built apparatus similar to that previously described. ${ }^{20,21}$ As shown schematically in Fig. 1, tissue culture plates containing the cell-polymer constructs in culture medium were placed in a rigid, cylindrical stainless steel pressure chamber. A pneumatically driven membrane chamber was used to create dynamic pressurization in the gas phase in the attached pressure chamber. Pressure transducers were placed in the gas phase to measure the actual pressure in the chamber. A digital data acquisition and analysis system with a sample frequency of $150 \mathrm{~Hz}$ was used to monitor the response. A $5 \% \mathrm{CO}_{2}$ level in the gas phase was maintained in the pressure chamber. Using this system, a cyclic, squarewave dynamic fluid pressure at a magnitude of $13 \mathrm{kPa}$ and a frequency of $0.3 \mathrm{~Hz}$ was applied to the constructs for $4 \mathrm{~h} /$ day.

\section{Sample analysis}

After 3 weeks and 6 weeks of culture, five chondrocyte-PGA constructs from each sample group (Static, S1/D5, S2/D4, and DFP) were harvested: three specimens for biochemical analysis, one for histologic evaluation, and one for scanning electron microscopy (SEM).

Biochemical analysis. The diameter and wet weight of the cell-polymer constructs were measured. The construct dry weight was obtained after drying with a SpeedVac (model SC200; Thermo Electron, Waltham, MA). A portion of the dried constructs was reserved for the collagentyping assay, whereas the rest was digested with a papain solution (Sigma, St. Louis, MO) as previously described ${ }^{22}$ for further biochemical analysis. Total DNA in the constructs was measured fluorometrically with a Hoechst 33258 (Polysciences, Warrington, PA) dye-binding assay. ${ }^{22}$ A conversion factor of 7.7 pg of DNA per chondrocyte was used to obtain the total cell number.

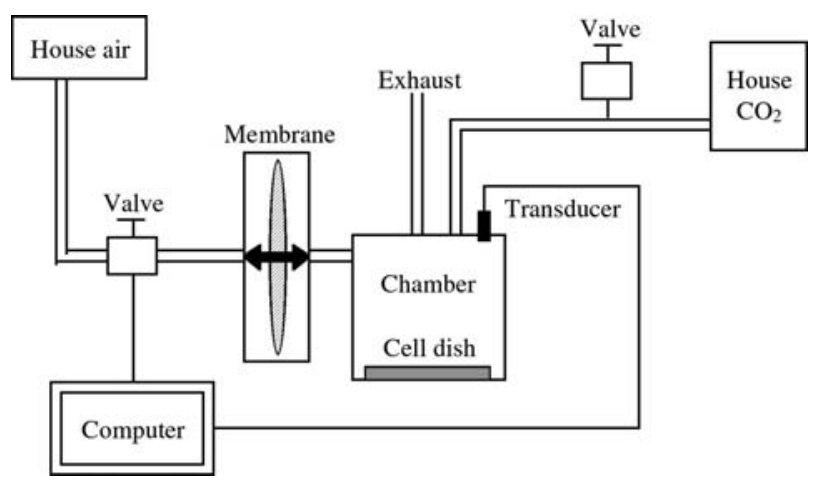

FIG. 1. Schematic of the dynamic fluid pressure system. 
Total collagen in the constructs was determined from the measured hydroxyproline content after acid hydrolysis, using a hydroxyproline-to-collagen ratio of 1:20.23 The percentage of type II collagen in the total collagen was quantified by comparing the spectrophotometric densities of specific cyanogen bromide peptide bands on sodium dodecyl sulfate-polyacrylamide gels with a standard curve. ${ }^{24}$ Glycosaminoglycan (GAG) content in the cellular constructs was determined by the dimethylene blue dye-binding assay. ${ }^{25}$

Histology. For histologic evaluation, the constructs were fixed in 10\% neutral buffered formalin (Sigma), dehydrated, and embedded in methyl methacrylate. Tissue blocks were sectioned with a microtome into $8-\mu \mathrm{m} \mathrm{sec-}$ tions and stained with safranin $\mathrm{O}$ for GAG distribution.

To quantify the relative amount of cartilage tissue present in the cell-polymer constructs, at least five sections obtained from various depths throughout the construct were analyzed with OsteoMeasure software (OsteoMetrics, Decatur, GA). The percentage of cartilage matrix was defined as the surface area stained red divided by the total surface area.

Scanning electron microscopy. The three-dimensional morphology of the cell-polymer constructs after 6 weeks of culture was assessed by SEM. On harvest, the constructs were washed with PBS, fixed in $2.5 \%$ glutaraldehyde (Sigma), and stained with $1 \%$ osmium tetroxide (Polysciences). The stained cell samples were dried in a critical point dryer, mounted on metal stubs with doublesided carbon tapes, and coated with a 50:50 mixture of gold and platinum. Both construct surfaces and freezefractured cross-sections were examined with a Hitachi S4700 field emission scanning microscope (Hitachi High Technologies America, Pleasanton, CA).

Statistical analysis. All data are reported as means \pm standard deviations (SD) for $n=3$ experiments, except for histologic quantification, where at least five sections were measured. Single-factor analysis of variance (ANOVA) was used to assess the statistical significance of results. The Scheffé method was employed for multiple comparison tests at significance levels of $95 \%$.

\section{RESULTS}

\section{Construct size, dry weight, and water content}

The size and weight of the chondrocyte-PGA constructs after 3 and 6 weeks of culture under Static, S1/D5, S2/D4, or DFP conditions were measured. The diameter of constructs was found to increase from $5.35 \pm 0.12$ $\mathrm{mm}$ for initial PGA disks to $6.73 \pm 0.27 \mathrm{~mm}$ after 6 weeks, indicating outward tissue growth from the scaffolds.

The dry weight of all cell-polymer constructs after incubation for 3 weeks was $4.33 \pm 0.25 \mathrm{mg}$, more than twice the initial weight of the scaffold $(1.70 \pm 0.27 \mathrm{mg})$, and nearly twice the weight of the non-cell-seeded polymer scaffolds incubated in culture medium $(2.23 \pm 0.42$ $\mathrm{mg}$ ). The slight increase was probably due to absorption by the scaffold of serum proteins in the culture medium. By 6 weeks in culture, the construct dry weight for all sample groups had increased to $6.34 \pm 0.16 \mathrm{mg}$. In contrast, PGA scaffolds without cells had completely disintegrated after 6 weeks and could not be retrieved for further evaluation.

The water content of the constructs, calculated from their wet and dry weights, was $88.7 \pm 1.5 \%$ for all sample groups after 3 or 6 weeks of culture. The water content of the constructs was in the range reported for native cartilage. ${ }^{26}$

\section{Cell density}

Cell number in the chondrocyte-PGA constructs was determined by the DNA assay and normalized to the construct dry weight (Fig. 2). All sample groups had similar $(p>0.05)$ cell densities at the two time points tested. By 6 weeks, the cell densities had increased to 106.7, 120.0, 108.1 , and $96.8\left(\times 10^{7}\right.$ per gram of construct dry weight $)$ for static, S1/D5, S2/D4, and DFP constructs, respectively. No significant $(p>0.05)$ increase in cell number from 3 to 6 weeks was found, except for the S1/D5 samples.

\section{Collagen}

Total collagen in the cell-polymer constructs was determined by hydroxyproline assay. Of the four sample groups studied, the collagen content as a percentage of construct dry weight was less than $10 \%$ after 3 weeks, but increased 2-fold after 6 weeks (Fig. 3A). Total collagen at 6 weeks $(23.7,20.2,19.0$, and $19.9 \%$ for the Static, S1/D5, S2/D4, and DFP groups, respectively) was about one-third that of native cartilage. ${ }^{26}$ Between 90 and $95 \%$ of the total collagen was type II collagen, as determined by collagen-typing assay (Fig. 3B). No significant $(p>0.05)$ difference between sample groups was observed. The percentage of collagen type II in the constructs was similar to that for normal cartilage. ${ }^{24}$

\section{GAG}

Glycosaminoglycan content as a percentage of construct dry weight was determined by dimethylene blue assay. As shown in Fig. 4, the GAG contents in the four sample groups were similar, increasing significantly $(p<$ 0.05 ) from approximately $8 \%$ at 3 weeks to $18 \%$ after 6 weeks. The values at 6 weeks were approximately half that for native cartilage. ${ }^{26}$ 


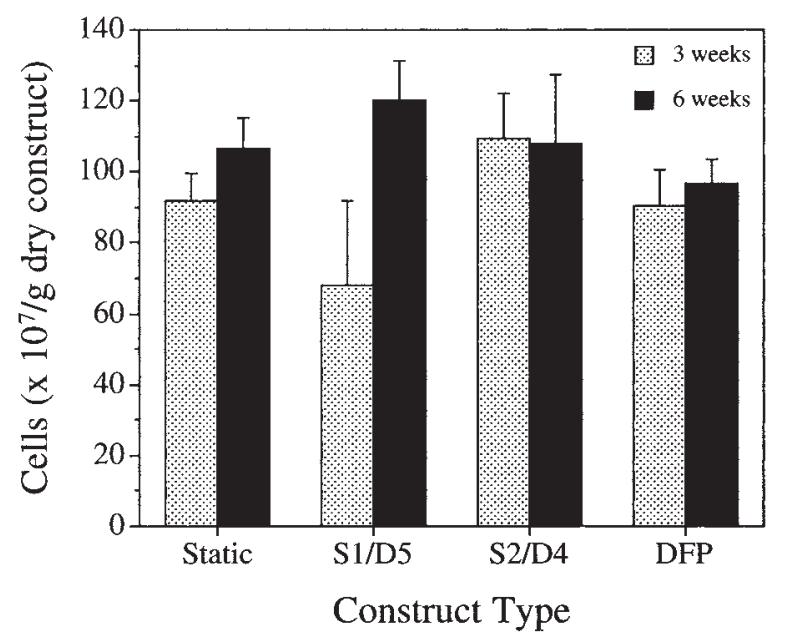

FIG. 2. Total cell number in chondrocyte-PGA constructs after 3 and 6 weeks of cultivation under Static, S1/D5, S2/D4, and DFP conditions. Static, cultured for 6 weeks under static conditions; S1/D5, 1 week of static culture and then 5 weeks under dynamic fluid pressure; S2/D4, 2 weeks of static culture and then 4 weeks under dynamic fluid pressure; DFP, 6 weeks under dynamic fluid pressure.

\section{Histology}

Chondrocyte-PGA constructs cultured under Static, S1/D5, S2/D4, and DFP conditions were stained with safranin $O$ for GAG distribution after 6 weeks. The periphery and middle portions of the constructs are shown, respectively, in the top and bottom panels of Fig. 5. Intense red staining at construct periphery indicated the production of a sulfated cartilage matrix for all sample groups (Fig. 5, top). The chondrocytes had a rounded morphology and resided inside lacunae. At the center of the constructs, however, staining was less intensive and polymer fibers not yet degraded were still present (Fig. 5 , bottom).

The relative amount of cartilage matrix in the tissue sections was quantified by normalizing the red-stained area to the whole section area. The results showed that between 94.8 and $97.9 \%$ of all cell-polymer constructs was cartilage matrix after 6 weeks.

\section{Morphology}

The three-dimensional morphology of the tissue formed in the PGA scaffolds was similar to that of native cartilage after 6 weeks. ${ }^{26}$ Figure 6 shows representative images of chondrocytes cultured for 2 weeks under static conditions followed by 4 weeks under DFP conditions (S2/D4 group). Chondrocytes were seen to cover the tissue surface (Fig. 6a and b); and the cells were spherical in shape and embedded in their extracellular matrix. A higher magnification view of the cross-sections shows a chondrocyte residing in its lacuna and surrounded by a highly fibrous extracellular matrix (Fig. 6c). A remaining PGA fiber was also seen adjacent to the cartilage matrix (Fig. 6d).

\section{DISCUSSION}

This study was designed to investigate the effects of dynamic fluid pressure on chondrocytes cultured in biodegradable PGA scaffolds. We investigated the effects of pressure application (at a magnitude of $13 \mathrm{kPa}$ and a frequency of $0.3 \mathrm{~Hz}$ ) as well as the time course of pressurization ( 4,5 , or 6 weeks with $4 \mathrm{~h}$ of pressurization per day).
A

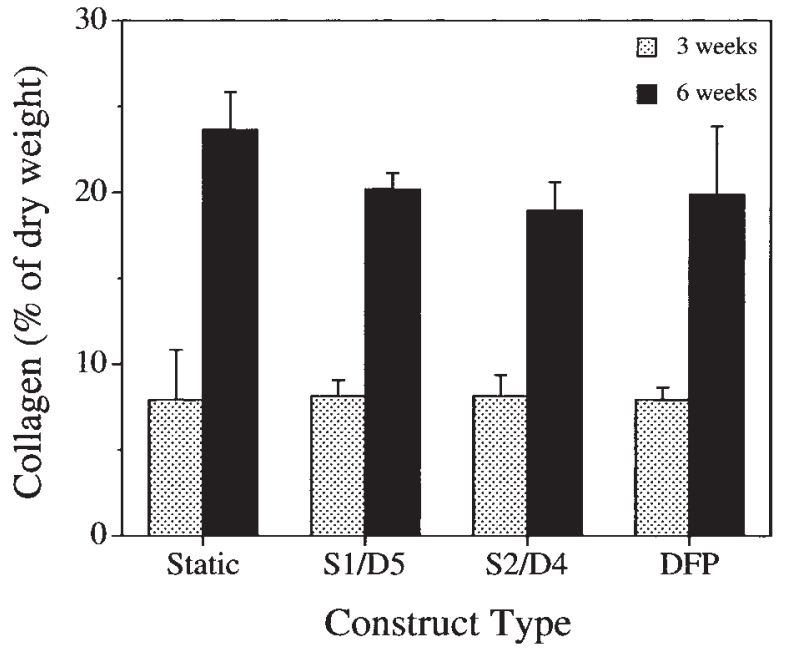

B

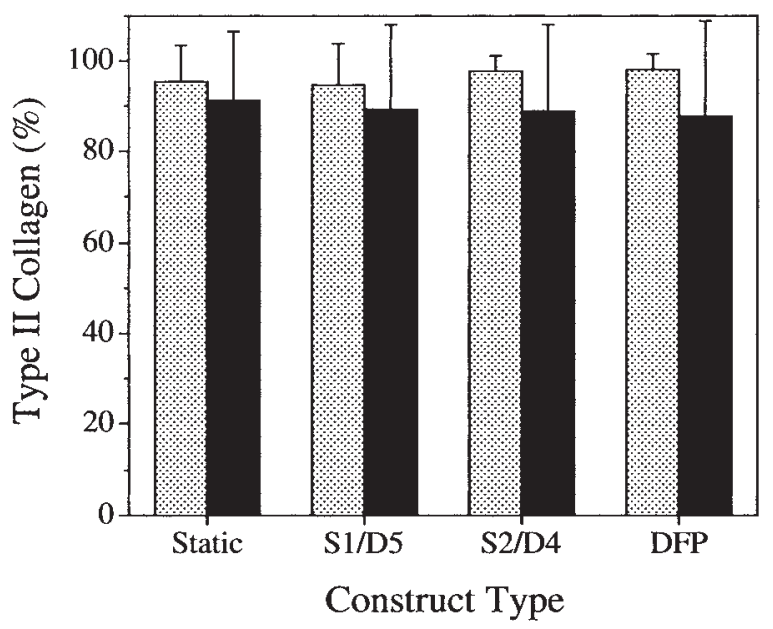

FIG. 3. (A) Total collagen and (B) percentage type II collagen relative to total collagen for chondrocyte-PGA constructs after 3 and 6 weeks of cultivation under Static, S1/D5, S2/D4, and DFP conditions. 


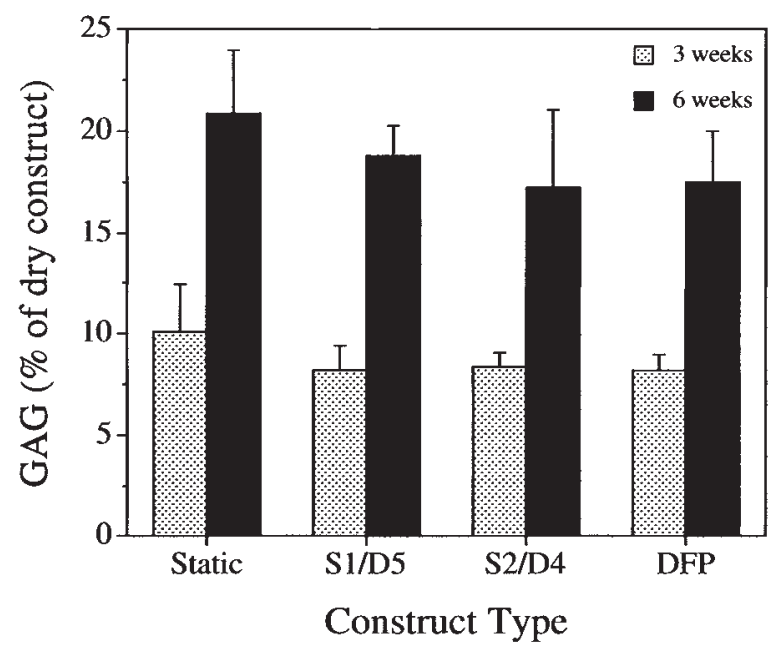

FIG. 4. Glycosaminoglycan (GAG) production in chondrocyte-PGA constructs after 3 and 6 weeks of cultivation under Static, S1/D5, S2/D4, and DFP conditions.

Cartilage matrices were developed in the chondrocytePGA constructs under all conditions studied. The water content in these constructs was about $90 \%$, similar to native cartilage. The dry constructs were composed of approximately $18 \%$ GAG and $20 \%$ collagen after 6 weeks of cultivation. Chondrocytes and remaining polymer fibers made up the majority of the rest of the construct dry weight. We showed that no significant difference in chondrocyte proliferation and cartilage matrix formation in the cellpolymer constructs was found between sample groups subjected to static culture (Static) or various time periods of dynamic fluid pressurization (S2/D4, S1/D5, and DFP).
The initial cell-seeding density used in this study was $5 \times 10^{6}$ cells per scaffold, corresponding to $300 \times 10^{7}$ cells/g of dry scaffold. Intermittent pressurization did not affect cell numbers compared with the static control (Fig. 2). Freed $e t a l .{ }^{26}$ seeded bovine calf articular chondrocytes in similar PGA fibrous disks at the same initial cell density. They observed that the construct cellularity reached a plateau within 6 days of culture in a rotating bioreactor, reaching $15 \times 10^{6}$ cells per construct. Our results also showed that cellularity leveled off between 3 and 6 weeks of culture for most sample groups. Carver and $\mathrm{Heath}^{27}$ used similar fibrous PGA mesh to seed foal articular chondrocytes and subjected the constructs to an intermittent hydrostatic pressure of $3.4 \mathrm{MPa}, 250$ times higher than the pressure used in our study. The cell numbers increased with culture times of 3 to 5 weeks but was not affected by pressurization. Therefore, our results confirm previous findings that cell numbers are not significantly affected by intermittent pressure. The cell number in our study after 6 weeks of culture with or without pressurization ranged between $10 \times 10^{7}$ and $12 \times 10^{7} \mathrm{cell} / \mathrm{g}$ wet weight, close to the native chondrocyte cell number of $4 \times 10^{7}$ to $6 \times 10^{7}$ cells/g wet weight. ${ }^{27}$

The total collagen percentage based on construct dry weight as a function of culture time is shown in Fig. 3A. No significant differences in collagen content were observed between the static and pressurized groups; however, the total collagen content increased by 3- and 2.7-fold for these groups, respectively, with increasing culture time from 3 to 6 weeks. It was also observed that total collagen content decreased slightly from $24 \%$ for static cultures to $20 \%$ for dynamic cultures. In previous studies, Carver and Heath ${ }^{27}$ found a 1.5 -fold increase in total collagen content

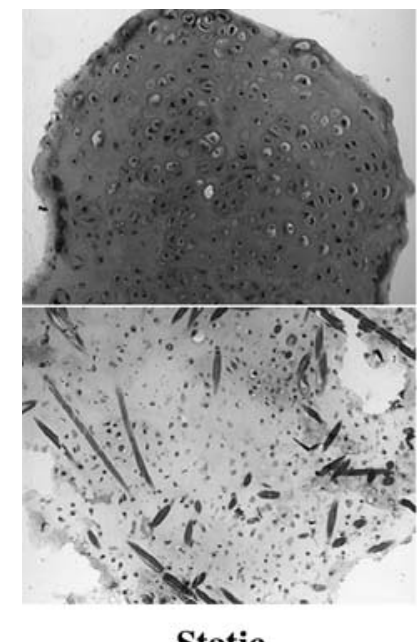

Static

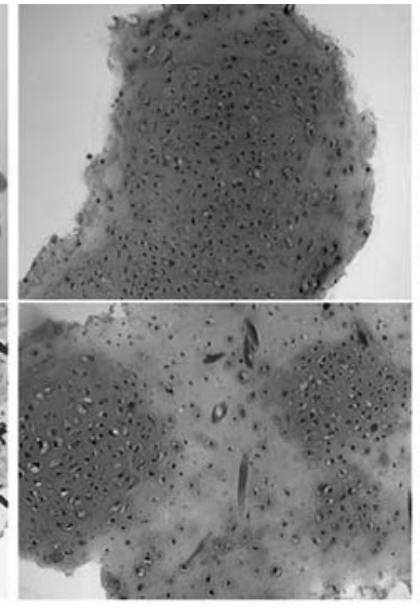

S1/D5

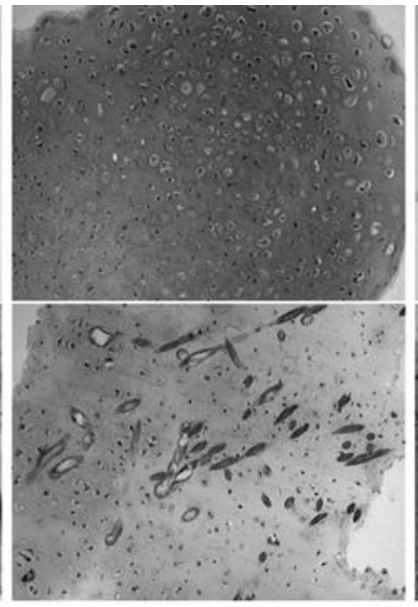

S2/D4

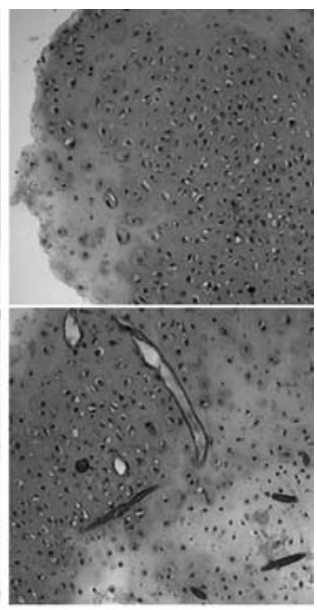

DFP

FIG. 5. Safranin O-stained histologic sections of chondrocyte-PGA constructs after 6 weeks of cultivation under Static, S1/D5, S2/D4, and DFP conditions. Both peripheral (top) and middle (bottom) sections of the constructs are shown. Original magnification, $\times 100$. 
(a)
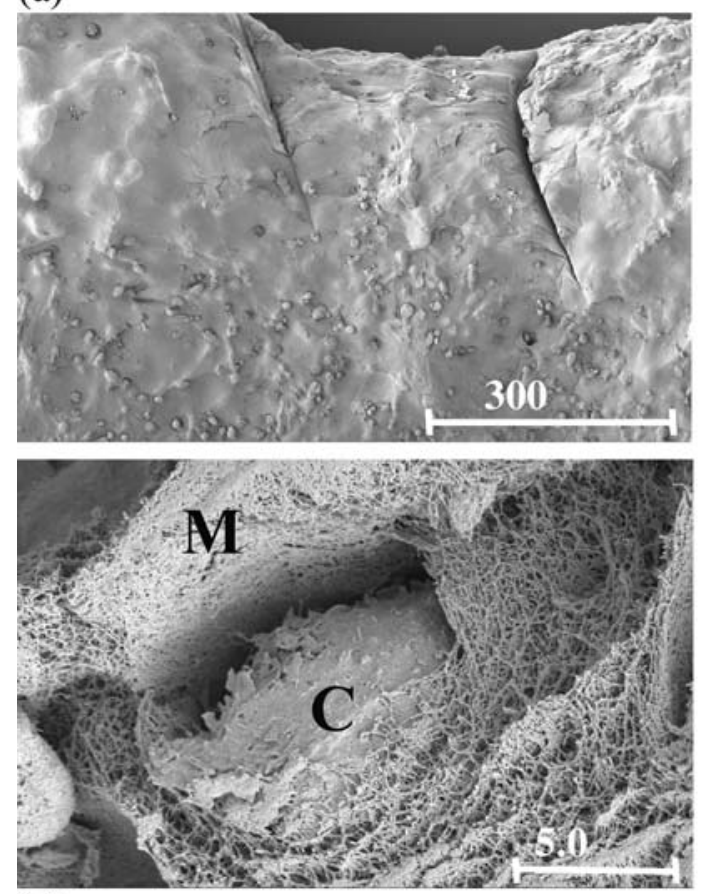

(c) (b)
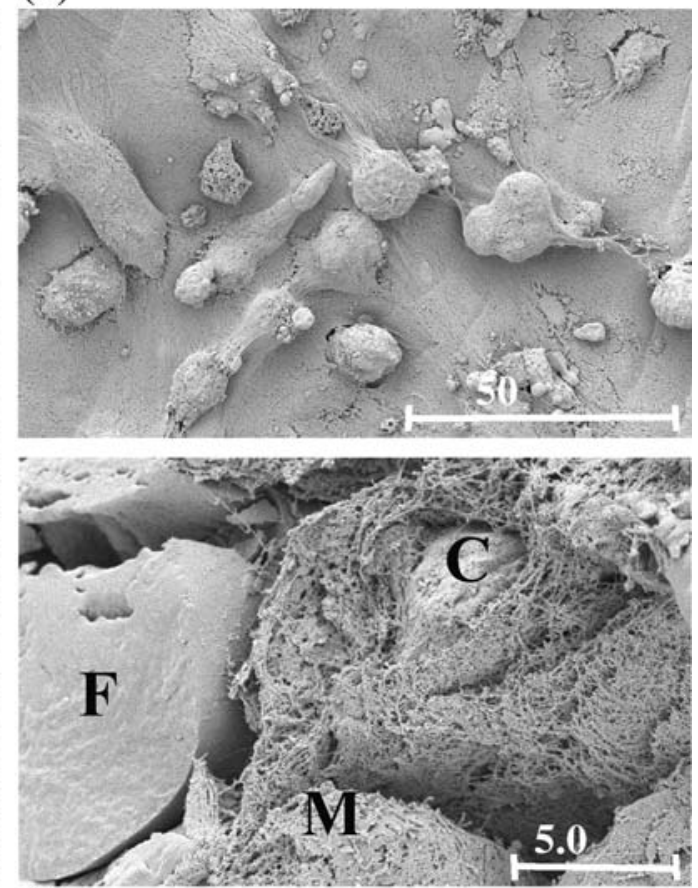

(d)

FIG. 6. Scanning electron micrographs of chondrocyte-PGA constructs cultured for 2 weeks under static conditions and then for 4 weeks under DFP conditions (S2/D4 samples). (a and b) Rounded chondrocytes were shown to cover the surface of the cartilage tissue. (c and d) Cross-sections showing the chondrocytes (C), the extracellular matrix (M), and a remaining PGA fiber (F). Scale bars: (a) $300 \mu \mathrm{m}$; (b) $50 \mu \mathrm{m}$; (c and d) $5 \mu \mathrm{m}$.

for foal chondrocytes after 5 weeks of culture with or without pressurization at 3.4 MPa. Moreover, they observed a slight decrease in total collagen for all time points when constructs were pressurized at $3.4 \mathrm{MPa}$, which is consistent with our findings with intermittent pressurization of 13 $\mathrm{kPa}$. In another experiment, enhanced collagen synthesis by equine chondrocytes in PGA scaffolds was found under an intermittent pressure of $6.8 \mathrm{MPa}$ but not $3.4 \mathrm{MPa},{ }^{28}$ suggesting that a high pressure level may be needed to stimulate collagen formation.

The percentage of type II collagen was higher than $90 \%$ for static as well as dynamic constructs after 3 and 6 weeks of culture (Fig. 3B). There was a slight (statistically insignificant) decrease in percent collagen type II from 95 to $91 \%$ when intermittent pressure was applied to the constructs. The finding of a high type II collagen content demonstrates that the chondrocyte phenotype was maintained in the constructs in the presence of a dynamic fluid pressure of $13 \mathrm{kPa}$. The percent type II collagen for dynamic constructs (91\%) was comparable to previously reported levels for chondrogenesis in a PGA bioreactor system in the absence of pressure ${ }^{26}$ and to the level for natural bovine articular cartilage $(90.3 \%) .{ }^{26}$ In another study, by Ikenoue et al., ${ }^{29}$ intermittent hydrostatic pressure was applied to normal human articular cartilage with pressure levels of 1,5 , and $10 \mathrm{MPa}$ for $4 \mathrm{~h} /$ day for either
1 day $(4 \times 1)$ or 4 days $(4 \times 4)$. They observed no change in expression pattern for collagen II for $4 \times 1$ loading regardless of the pressure level. However, the collagen II expression level was upregulated with $4 \times 4$ regimens only for pressure levels of 5 and $10 \mathrm{MPa}$. Therefore, consistent with our findings, collagen II production is not influenced by a short duration or low magnitude of intermittent hydrostatic pressure.

The GAG content in the constructs approximately doubled as the culture time was increased from 3 to 6 weeks (Fig. 4). A slight (statistically insignificant) decrease in GAG content from $21 \%$ of dry construct for static cultures to $16-18 \%$ of dry construct for dynamic cultures was observed. The GAG content after 6 weeks of culture with intermittent pressure was $18 \%$ of dry mass, which was significantly less than that of native articular cartilage (41\%). ${ }^{26}$ Carver and Heath ${ }^{27}$ found that an intermittent pressure of 3.4 MPa significantly increased GAG content, exhibiting native GAG levels after 6 weeks.

As pointed out in introduction, we chose a pressure magnitude of $13 \mathrm{kPa}$ and a pressure frequency of $0.3 \mathrm{~Hz}$ to mimic the normal component of the fluid pressure during continuous passive motion. This pressure regimen has been shown previously to stimulate cellular activity in cultured periosteal cells. ${ }^{20}$ However, our results indicate that an intermittent hydrostatic pressure of $13 \mathrm{kPa}$ has no 
effect on cellularity, total collagen, and GAG content of the chondrocytes cultured in PGA scaffolds. Analysis of joint loading profiles shows that mechanical forces that produce either dilational or deviatoric stresses exhibit differential effects on articular chondrocyte metabolism. The loading mode in this study was normal hydrostatic pressure with no direct deformation or fluid flow or deformation-induced shear stresses transmitted to the seeded chondrocytes. Future experiments are required to investigate the effects of direct deformation and fluid shear, in combination with dynamic fluid pressure, in order to further elucidate the effects of continuous passive motion on chondrogenesis of cell-PGA constructs.

\section{CONCLUSIONS}

We have shown that cartilage matrices were produced in chondrocyte-PGA constructs after 6 weeks of culture. The amount of type II collagen and glycosaminoglycan in the dry constructs increased significantly from 3 to 6 weeks. The formed matrices were similar to normal cartilage in terms of water content and percentage of type II collagen. We also found that the normal component of the hydrostatic force did not affect cartilage matrix formation under the conditions tested. To further elucidate the role of continuous passive motion, more experiments are needed to study the other forces transmitted to cartilage, such as direct deformation and fluid shear, or the combined effects of dynamic fluid pressure with these forces.

\section{ACKNOWLEDGMENTS}

This work was supported by the Mayo Foundation, the John Smith Foundation, and the Aircast Foundation. The authors thank Dr. Gordana Vunjak-Novakovic at the Massachusetts Institute of Technology for assistance with the biochemical assays.

\section{REFERENCES}

1. National Center for Chronic Disease Prevention and Health Promotion, Centers for Disease Control and Prevention (CDC). Data available at http://www.cdc.gov/nccdphp/aag/ aag_arthritis.htm (accessed November 2005).

2. Lu, L., Valenzuela, R.G., and Yaszemski, M.J. Articular cartilage tissue engineering. e-biomed 2, 99, 2000.

3. LeBaron, R.G., and Athanasiou, K.A. Ex vivo synthesis of articular cartilage. Biomaterials 21, 2575, 2000.

4. Lu, L., Zhu, X., Valenzuela, R.G., Currier, B.L., and Yaszemski, M.J. Biodegradable polymer scaffolds for cartilage tissue engineering. Clin. Orthop. 391, S251, 2001.

5. Temenoff, J.S., and Mikos, A.G. Review: Tissue engineering for regeneration of articular cartilage. Biomaterials 21, 431, 2000.
6. Elisseeff, J., Anseth, K., Sims, D., McIntosh, W., Randolph, M., and Langer, R. Transdermal photopolymerization for minimally invasive implantation. Proc. Natl. Acad. Sci. U.S.A. 96, 3104, 1999.

7. Anseth, K.S., Shastri, V.R., and Langer, R. Photopolymerizable degradable polyanhydrides with osteocompatibility. Nat. Biotechnol. 17, 156, 1999.

8. Temenoff, J.S., Athanasiou, K.A., Lebaron, R.G., and Mikos, A.G. Effect of poly(ethylene glycol) molecular weight on tensile and swelling properties of oligo(poly(ethylene glycol) fumarate) hydrogels for cartilage tissue engineering. J. Biomed. Mater. Res. 59, 429, 2002.

9. Freed, L.E., Grande, D.A., Lingbin, Z., Emmanual, J., Marquis, J.C., and Langer, R. Joint resurfacing using allograft chondrocytes and synthetic biodegradable polymer scaffolds. J. Biomed. Mater. Res. 28, 891, 1994.

10. Grande, D.A., Halberstadt, C., Naughton, G., Schwartz, R., and Manji, R. Evaluation of matrix scaffolds for tissue engineering of articular cartilage grafts. J. Biomed. Mater. Res. 34, 211, 1997.

11. Schreiber, R.E., Ilten-Kirby, B.M., Dunkelman, N.S., Symons, K.T., Rekettye, L.M., Willoughby, J., and Ratcliffe, A. Repair of osteochondral defects with allogeneic tissue engineered cartilage implants. Clin. Orthop. Relat. Res. 367(Suppl.), S382, 1999.

12. Vunjak-Novakovic, G., Martin, I., Obradovic, B., Treppo, S., Grodzinsky, A.J., Langer, R., and Freed, L.E. Bioreactor cultivation conditions modulate the composition and mechanical properties of tissue-engineered cartilage. J. Orthop. Res. 17, 130, 1999.

13. Martin, I., Obradovic, B., Treppo, S., Grodzinsky, A.J., Langer, R., Freed, L.E., and Vunjak-Novakovic, G. Modulation of the mechanical properties of tissue engineered cartilage. Biorheology 37, 141, 2000.

14. Guilak, F., Butler, D.L., and Goldstein, S.A. Functional tissue engineering: The role of biomechanics in articular cartilage repair. Clin. Orthop. 391(Suppl.), S295, 2001.

15. O'Driscoll, S.W. The healing and regeneration of articular cartilage. J. Bone Joint Surg. Am. 80, 1795, 1998.

16. O'Driscoll, S.W., Kumar, A., and Salter, R.B. The effect of the volume of effusion, joint position and continuous passive motion on intraarticular pressure in the rabbit knee. J. Rheumatol. 10, 360, 1983.

17. Lafeber, F., Veldhuijzen, J.P., Vanroy, J.L., Huber-Bruning, O., and Bijlsma, J.W. Intermittent hydrostatic compressive force stimulates exclusively the proteoglycan synthesis of osteoarthritic human cartilage. Br. J. Rheumatol. 31, 437, 1992.

18. Brittberg, M., Nilsson, A., Lindahl, A., Ohlsson, C., and Peterson, L. Rabbit articular cartilage defects treated with autologous cultured chondrocytes. Clin. Orthop. Relat. Res. 326, 270, 1996.

19. Schreiber, R.E., Dunkelman, N.S., Naughton, G., and Ratcliffe, A. A method for tissue engineering of cartilage by cell seeding on bioresorbable scaffolds. Ann. N.Y. Acad. Sci. 875, 398, 1999.

20. Saris, D.B., Sanyal, A., An, K.N., Fitzsimmons, J.S., and O'Driscoll, S.W. Periosteum responds to dynamic fluid pressure by proliferating in vitro. J. Orthop. Res. 17, 668, 1999. 
21. Saris, D.B., Mukherjee, N., Berglund, L.J., Schultz, F.M., An, K.N., and O'Driscoll, S.W. Dynamic pressure transmission through agarose gels. Tissue Eng. 6, 531, 2000.

22. Kim, Y.-J., Sah, R.L.Y., Doong, J.-Y.H., and Grodzinsky, A.J. Fluorometric assay of DNA in cartilage explants using Hoechst 33258. Anal. Biochem. 174, 168, 1988.

23. Woessner, J.F., Jr. The determination of hydroxyproline in tissue and protein samples containing small proportions of this imino acid. Arch. Biochem. Biophys. 93, 440, 1961.

24. O'Driscoll, S.W., Salter, R.B., and Keeley, F.W. A method for quantitative analysis of ratios of types I and II collagen in small samples of articular cartilage. Anal. Biochem. 145, 277, 1985.

25. Farndale, R.W., Buttle, D.J., and Barrett, A.J. Improved quantitation and discrimination of sulphated glycosaminoglycans by use of dimethylmethylene blue. Biochim. Biophys. Acta 883, 173, 1986.

26. Freed, L.E., Hollander, A.P., Martin, I., Barry, J.R., Langer, R., and Vunjak-Novakovic, G. Chondrogenesis in a cellpolymer-bioreactor system. Exp. Cell Res. 240, 58, 1998.

27. Carver, S.E., and Heath, C.A. Semi-continuous perfusion system for delivering intermittent physiological pressure to regenerating cartilage. Tissue Eng. 5, 1, 1999.

28. Carver, S.E., and Heath, C.A. Increasing extracellular matrix production in regenerating cartilage with intermittent physiological pressure. Biotechnol. Bioeng. 62, $166,1999$.

29. Ikenoue, T., Trindade, M.C., Lee, M.S., Lin, E.Y., Schurman, D.J., Goodman, S.B., and Smith, R.L. Mechanoregulation of human articular chondrocyte aggrecan and type II collagen expression by intermittent hydrostatic pressure in vitro. J. Orthop. Res. 21, 110, 2003.

Address reprint requests to: Michael J. Yaszemski, M.D., Ph.D. Orthopedic Surgery and Biomedical Engineering Mayo Clinic College of Medicine 200 1st Street SW Rochester, MN 55902-0002

E-mail: yaszemski.michael@mayo.edu 